narrow and wide reversal respectively was not desirable for astronomical spectra, in which both absorption and emission lines might often be found together, or even the same line appear in both absorption and emission. Prof. Harrison felt that this would not cause confusion, since $r$ and $R$ were to be taken as indicating absorption in an emission line, or emission in an absorption line. Specific statement would in any case be needed to indicate whether the main portion of the line appeared in absorption or in emission. The recommendation was adopted as given in the Draft Report on p. Ior, with the single change mentioned above regarding the symbol $\mathrm{A}$.

\title{
COMMISSION I5 (PHYSICAL STUDY OF THE COMETS)
}

President: Dr F. Baldet.

Secretary: Miss Helen W. Dodson.

En ouvrant la séance le Président rappelle que la Commission I5, créée en I935, se réunit pour la première fois. Il rend hommage à la mémoire du Comte A. de la Baume Pluvinel, Président du Comité National Français d'Astronomie, récemment décédé, qui devait participer aux travaux de la Commission. Cet astronome avait beaucoup étudié les spectres des comètes et c'est à lui qu'on doit, en particulier, la première application réussie (I902) du prisme-objectif à leur étude.

Le Président fournit quelques explications sur le rapport préliminaire qui donne un résumé des travaux effectués sur la physique des comètes depuis trois ans. II s'est proposé de montrer où en étaient les questions qui vont faire l'objet des discussions de la Commission afin de pouvoir mieux orienter une collaboration internationale. Le rapport est adopté sans modifications.

Il dépose sur le bureau les exemplaires dactylographiés, traduits du russe en français, de six mémoires du Prof. Orlov sur la théorie des comètes. Ces traductions sont à la disposition des membres qui désireraient en prendre connaissance.

Les trois suggestions publiées aux "Draft Reports" (p. II3) sont mises en discussion. En ce qui concerne la première, M. Dufay rappelle que des échanges de photographies de la comète Finsler (I937f) ont déjà eu lieu à la demande du regretté Ellsworth de l'Observatoire de Lyon, qui désirait entreprendre une étude des mouvements des nébulosités observées dans la queue de cette comète. Le Président a envoyé à l'Observatoire de Zurich une série des photographies de cette même comète prises à l'Observatoire de Meudon.

Les deux autres suggestions sont soumises à discussion et des échanges de vue sur la photométrie des comètes par images extra-focales ont lieu.

M. Vandekerkhove attire l'attention sur les avantages que présente la chambre de Schmidt à grande ouverture relative dans l'étude photographique des comètes et l'intérêt qu'il y aurait à en répandre l'usage.

Le Président lit une note communiquée par $M$. Delporte sur les travaux concernant les observations de comètes et la théorie de leur origine effectués à l'Observatoire d'Uccle.

La Commission adopte les quatre résolutions suivantes:

(I) La Commission recommande de publier une liste des photographies des comètes et de leurs spectres classées par ordre chronologique. La publication de cette liste sera faite trimestriellement, et à titre d'essai, aux Annales de l'Observatoire de Meudon par les soins du Président. 
Un appel sera adressé aux observatoires convenablement situés en longitude pour aider à combler les lacunes importantes que présentent actuellement les séries d'observations.

(2) La Commission attire l'attention des observateurs sur la nécessité d'unifier les déterminations des magnitudes photographiques et visuelles des comètes afin de les rendre comparables entre elles. Elle les engage à approfondir la question de manière à pouvoir confronter les méthodes et choisir la meilleure, dans chaque cas, au prochain Congrès.

La même recommandation est faite pour la spectrophotométrie des comètes.

(3) La Commission n'a pas la possibilité matérielle d'entreprendre elle-même la création d'un "Bureau des Comètes" et d'en assurer le fonctionnement. Mais elle encouragera tout observatoire ou astronome qui se chargerait de la discussion des observations obtenues sur une ou plusieurs comètes en lui facilitant l'envoi des documents photographiques dont il aura trouvé la liste dans la publication prévue au paragraphe (I).

(4) L'emploi de systèmes optiques à grand rapport d'ouverture ( $F / I$ par exemple) tels que la chambre de Schmidt est particulièrement recommandé pour l'étude photographique des comètes.

Une seconde séance est spécialement consacrée à la spectrophotométrie des cometes.

Le Dr Swings présente de nouveaux travaux sur cette question et les conclusions qu'il en a tirées. Les bandes cométaires offrant des répartitions d'intensités différentes de celles qu'on observe en laboratoire, surtout dans les termes de rotation, il y aurait lieu de reprendre certaines identifications sur de meilleures données d'observation. Pour ce genre de recherches, les spectres de comètes doivent être obtenus avec des spectrographes à fente. Il expose ses idées concernant la position des maxima d'intensité dans les bandes cométaires. Le détail de ce travail paraîtra prochainement à l'Astrophysical Journal.

M. Dufay présente les résultats qu'il a obtenus récemment en dépouillant au microphotomètre les spectres des dernières comètes photographiés à l'Observatoire de Lyon. Ils donnent des résultats en accord avec ceux prévus par le Dr Swings.

Le Président attire l'attention sur les incertitudes que présente parfois l'interprétation des tracés microphotométriques des spectres cométaires si étroits donnés par le prisme-objectif. Il demande que les raies décelées par superposition des tracés, mais invisibles par simple examen du cliché à la machine à mesurer, soient signalées dans les publications au moyen d'un signe convenable.

Le Prof. Végard pose une série de questions concernant les caractéristiques des spectres que M. Swings a utilisés pour ses recherches et les méthodes qui ont été employées pour la mesure des intensités.

La Commission proposera MM. Dufay, Swings et Bourgeois comme nouveaux membres. 